\section{Defective yet strong} Nature Commun. 5, 3186 (2014)

With a tensile strength of over $100 \mathrm{GPa}$, a pristine graphene layer is the strongest material known. However, most graphene fabrication processes inevitably produce a defective layer. Also, structural defects are desirable in some of the material's technological applications, as defects allow for the opening of a bandgap or act as pores for molecular sieving, for example. Now, by tuning the exposure of graphene to oxygen plasma to control the creation of defects, Ardavan Zandiatashbar et al. show that a single layer of graphene bearing $s p^{3}$-type defects (chemisorbed oxygen atoms) at a high density (an average spacing between defects of $5 \mathrm{~nm}$ ) maintains its stiffness, and that its breaking strength is only reduced by $14 \%$. Instead, the presence of vacancies (formed by the removal of carbon atoms at higher oxygen-plasma exposure) causes both mechanical properties to degrade significantly from those of the pristine layer. The researchers also show that Raman spectra can be mapped to the measured stiffness and strength, thereby providing a non-destructive method to predict these properties from the respective Raman parameters.

\section{Quasi-Dirac monopoles}

Nature 505, 657-660 (2014)
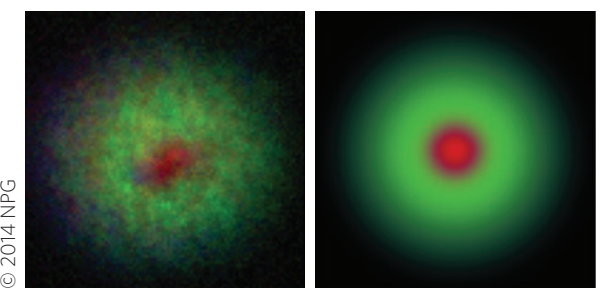

Since the publication of Dirac's classical paper in 1931, the search for a new fundamental particle, the magnetic monopole, with non-zero magnetic monopole charge, has grasped the attention of many. The existence of magnetic monopoles is now not only supported by the work of Dirac, but by both the grand unified theory and string theory. However, both theories realize that magnetic monopoles might be too few and too massive to be detected or created in a lab, so physicists started to explore approaches to obtain such particles using condensed-matter systems. Now, Ray and co-workers report the latest observation of a Dirac monopole quasiparticle (pictured), this time in a spinor Bose-Einstein condensate. This magnetic monopole quasiparticle is in fact not a magnetic monopole fundamental particle, but a quasiparticle equivalent, and is a source of a 'synthetic magnetic field' that violates the zero-divergence law. Even though this system only resembles a true magnetic monopole, this is the first time such a quasiparticle has been detected within a system governed by quantum field theory, the framework behind subatomic particle physics. ON

\section{Plasmons on screen Nature Commun. 5, 3152 (2014)}

See-through displays are increasingly used in aviation and automotive applications, as well as in gaming and entertainment. The most common technology used to fabricate them relies on transparent organic light-emitting diodes, but cheaper and more scalable alternatives based on light-responsive elements dispersed in a glassy or polymeric matrix are being studied at present. Following the latter approach, Chia Wei Hsu and colleagues now realize a transparent screen that displays bright, blue images projected onto it from a commercial laser projector. In a

\title{
Motorizing graphene fibres
}

The practical potential of graphene fibres (GFs) stems from the fact they combine the mechanical strength and flexibility of fibres with the electronic and thermal characteristics of graphene. Recently, a spinning technique for fabricating GFs directly from graphene oxide has galvanized research in this area, as this provides a large-scale and low-cost route to fibres with a range of different functionalities that one can envisage being useful in textiles for wearable electronics. Now, Liangti Qu and colleagues from the Beijing Institute of Technology demonstrate an intriguing concept for GFs, in the form of moisture-operated twisting graphene motors. Depending on the degree of twisting they are subjected to, different twist angles can be introduced in the GFs. Once a particular twist angle has been set, it can be reversibly tuned by exposing the fibres to moisture. The authors then make use of this property to fabricate an electric generator in which the application of vapour leads to a twisted GF switching the magnetization of a small magnet, which in turn drives a small electric field in copper coils surrounding it.

polymeric film, the researchers embedded silver nanoparticles that have a localized surface plasmon resonance in the blue spectral range; the fabricated film therefore scatters this colour while being almost completely transparent in the remaining visible spectral range. The researchers suggest that dispersion in a single matrix of metallic nanoparticles with sharp plasmonic resonances tuned at different wavelengths will lead to the realization of multicoloured transparent displays.

\section{Facet formation}

Adv. Mater. http://doi.org/rb5 (2014)

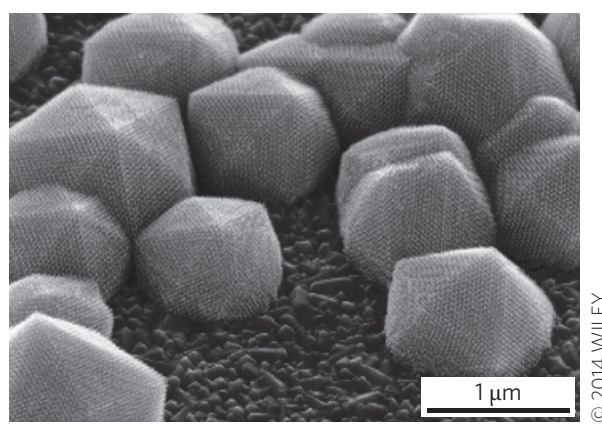

Crystal facets result from anisotropic growth along different crystallographic directions and, as a consequence, do not usually exist for polycrystalline materials that have no long-range order and grow isotropically. Now, Ullrich Steiner and colleagues show that faceted platinum particles (pictured), which are inherently polycrystalline, can be isolated following electrochemical synthesis within mesoporous polymeric templates with double-gyroid morphology. Without such a template, spherical platinum particles are produced. Faceted platinum particles with well-defined symmetries are made when the template has large doublegyroid monodomains, however, if the double-gyroid morphology has randomly orientated multi-domains, an irregularly faceted particle is formed. The particles made within the monodomain templates are tetrahexahedral in shape, with 24 equal faces. Steiner and colleagues use a numerical model to explain the formation of the faceted particles and with the aid of microscopic analysis, report a nucleationcoalescence mechanism in which the growth front progresses along the centre of the channels of the template. This causes a slowing of growth in all directions - in particular, in the 110 direction, yielding the tetrahexahedra observed.

Written by Luigi Martiradonna, Olivia Nicoletti, Pep Pàmies, Alison Stoddart and Andrea Taroni. 